\title{
Interventions to prevent and treat short-term and long-term nutritional problems in childhood cancer: A systematic review
}

\author{
L. Stewart, M. Wilson and D. C. Wilson \\ Child Life and Health, University of Edinburgh, Edinburgh EH9 1 UW
}

Nutritional problems are common in children with cancer. Undernutrition at diagnosis is associated with a worsened outcome, development of undernutrition is common during treatment of cancer, yet obesity is a frequent long-term consequence in survivors of childhood cancer. We aimed to ascertain the effectiveness of interventions to prevent or treat these problems in children with cancer by systematic review.

A computer-assisted search of the literature for nutritional interventions to prevent or treat short-term undernutrition and long-term obesity in children with cancer was performed using the following on-line databases: MEDLINE (1966-June 2006), EMBASE (1980June 2006), CINAHL (1983-June 2006) and the Cochrane Library.

There were neither any evidence-based clinical guidelines nor any systematic reviews of nutritional interventions in childhood cancer. We found no good quality randomised controlled trial of prevention or treatment of either short-term undernutrition or long-term obesity in children with cancer. There is a lack of an evidence base to guide early nutrition support or long-term strategies to prevent obesity in children with cancer.

Those delivering care should closely monitor nutritional status during and post treatment. Well-conducted research investigating factors which may compromise nutrition during treatment and lead to obesity long-term should be undertaken. 